\title{
No Tilt Angle Dependence of Grain Boundary on Mechanical Strength of Chemically Deposited Graphene Film
}

\author{
Jong Hun Kim**, Sung Joo An***, Jong-Young Lee $* * * *$ Eunji Ji***, \\ James Hone**, and Gwan-Hyoung Lee ${ }^{(1)} * \dagger$ \\ *Department of Materials Science and Engineering, Seoul National University, Seoul 08826, Korea \\ **Department of Mechanical Engineering, Columbia University, New York 10027, USA \\ ***Department of Materials Science and Engineering, Yonsei University, Seoul 03722, Korea
}

(Received August 22, 2019; Revised September 12, 2019; Accepted September 16, 2019)

\begin{abstract}
Although graphene has been successfully grown in large scale via chemical vapor deposition (CVD), it is still questionable whether the mechanical properties of CVD graphene are equivalent to those of exfoliated graphene. In addition, there has been an issue regarding how the tilt angle of the grain boundary (GB) affects the strength of graphene. We investigate the mechanical properties of CVD graphene with nanoindentation from atomic force microscopy and transmission electron microscopy. Surprisingly, the samples with GB angles of $10^{\circ}$ and $26^{\circ}$ yielded similar fracture stresses of $\sim 80$ and $\sim 79 \mathrm{GPa}$, respectively. Even for samples with GB exhibiting a wider range, from $0^{\circ}$ to $30^{\circ}$, only a slightly wider fracture stress range $(\sim 50$ to $\sim 90 \mathrm{GPa})$ was measured, regardless of tilt angle. The results are contrary to previous studies that have reported that GBs with a larger tilt angle yield stronger graphene film. Such a lack of angle dependence of GB can be attributed to irregular and well-stitched GB structures.
\end{abstract}

\section{Key words : CVD-graphene, Polycystalline Graphene, Grain boundary, Stress, Nano indentation}

\section{Introduction}

$\mathrm{D}$ efect-free exfoliated graphene is reportedly the strongest material in the world, exhibiting high elastic stiffness. ${ }^{1-4)}$ Despite this amazing feature, exfoliated graphene has limits in mass production. Thus, graphene film, grown by the chemical vapor deposition (CVD) process, has been considered as the most promising alternative. Because polycrystalline graphene is inevitably synthesized in CVD graphene, it is important to understand how defects accommodate the local strain and lattice mismatch between graphene grains with different crystalline orientations, ${ }^{5-9}$ as graphene occasionally loses carbon atoms in point defects, such as vacancies, voids, and Stone-Wales (SW) defects. ${ }^{10)}$ Because a $\mathrm{C}-\mathrm{C}$ bond needs to rotate by $90^{\circ}$ from the bonding center to create SW defects, four hexagons are converted to two pentagons and two heptagons. Therefore, the grain boundary (GB) strength is determined by the detailed arrangement as well as the defect density. When the pentagon-heptagon defects are assumed to be distributed homogenously at the $\mathrm{GB}$, the strength of the tilted GBs increases with the square of the tilt angle. Furthermore, a high-tilt-angle GB with

\footnotetext{
¥These authors contributed equally.

${ }^{\dagger}$ Corresponding author: Gwan-Hyoung Lee

E-mail : gwanlee@snu.ac.kr

Tel : +82-2-880-8366 Fax : +82-2-885-9671

ORCID

https://orcid.org/0000-0002-3028-867X
}

denser dislocations is computed to show higher strength, contrary to conventional bulk materials for which strength declines with defect density. ${ }^{11)}$ In addition, the loss of mechanical strength in CVD graphene is claimed to be much smaller than expected, as the complicated atomic bonding structures of GBs can efficiently relax the applied strain. ${ }^{7,11,12)}$ Furthermore, some theoretical and experimental studies have shown that the mechanical strength of CVD graphene is almost equivalent to that of exfoliated graphene, despite GBs and other defects such as vacancies. ${ }^{7,13-15)}$

Despite the fact that some theoretical calculations show the possibility of producing large-scale polycrystalline graphene as strong as pristine graphene, continuous debate regarding the true correlation between the mechanical deterioration of CVD graphene and the misorientation angles at the GB has persisted. ${ }^{11-13)}$ Realizing the relatively high mechanical strength of CVD graphene will shed light on the practical applications of CVD graphene. ${ }^{4,16,17)}$

In this paper, we aimed to present the angular dependence of the mechanical properties of grains and GBs. Furthermore, we investigated the crack propagation at the rupture of graphene after nanoindentation. The results demonstrate that the mechanical strength of CVD graphene with well-stitched and complex GBs has no correlation with the tilt angle. 


\section{Experimental Procedure}

\subsection{Sample preparation}

Figure 1 shows the sample preparation process and graphene transfer process. For the growth of CVD graphene, the copper foil was heated to $1000^{\circ} \mathrm{C}$ in a hydrogen flow of 2 sccm at a pressure of $50 \mathrm{~m}$ Torr. After annealing for $60 \mathrm{~min}$, graphene was grown by introducing methane gas flow of 35 sccm while maintaining hydrogen flow. After growth at 300 $\mathrm{m}$ Torr and $1000^{\circ} \mathrm{C}$ for $30 \mathrm{~min}$, the sample was rapidly cooled to ambient temperature under a flow of methane and hydrogen to achieve polycrystalline graphene, with grain sizes ranging from 1 to $5 \mu \mathrm{m}$. For the atomic force microscopy (AFM) nanoindentation test, the synthesized CVD graphene film was transferred onto the substrates with holes of radius $1.5 \mu \mathrm{m}$ or onto a silicon nitride transmission electron microscopy (TEM) grid with holes of radius $2.5 \mu \mathrm{m}$, as we previously reported. ${ }^{1,7)}$ By using the polymethyl methacrylate (PMMA) transfer technique, ${ }^{18,19)}$ CVD graphene film was transferred on top of the substrate. After coating the graphene/Cu-foil stack with the PMMA layer, the copper was completely etched with ammonium persulfate for 2 h. After repetitive rinsing processes with DI water, the floating PMMA-coated graphene sheet was scooped with a TEM grid. After complete drying, the PMMA layer was removed by dipping the sample in acetone for $10 \mathrm{~min}$ and annealing at $345^{\circ} \mathrm{C}$ for $1 \mathrm{~h}$ in a gas mixture of argon and hydrogen. To prevent the formation of cracks and failure, care should be taken to reduce the excessive surface tension when the samples are taken out from the solution.

\subsection{Characterization methods}

The location of the suspended graphene film was identi- fied with optical microscopy and AFM (Park Systems, XE100). To determine the quality of the suspended graphene films, Raman spectroscopy (Renishaw, inVia) was employed with a 532-nm laser. TEM (JEOL JEM-100CX) was used for the observation of GBs. We used a low operating voltage of $80 \mathrm{kV}$ to minimize any possible damage to graphene caused by electron bombardment. To measure the mechanical strength of CVD graphene, AFM (Park Systems, XE-100) was used with a diamond AFM tip.

\section{Results and Discussion}

Before the mechanical test of CVD graphene via AFM nanoindentation, ${ }^{4,20)}$ we confirmed that CVD graphene is transferred and suspended on the TEM grid via TEM, as shown in Fig. 2(a) and (b). The CVD graphene film was successfully transferred onto the TEM grid without any cracks or damage. However, PMMA residue (dark spots on the suspended graphene region) was observed in all the transferred graphene samples. The non-contact AFM topography and phase images of the suspended CVD graphene samples in Fig. 2(c) and (d) show that the suspended graphene is stable during scanning, and there are no cracks or damage except the PMMA residue (indicated by blue arrows in Fig. 2(c)). Thus, it can be concluded that the graphene transfer process leads to the absence of crack formation, and the suspended graphene samples for mechanical tests can be successfully fabricated. The PMMA residue cannot be removed completely even after annealing. However, it is interesting that most of the PMMA residue is located primarily along the GBs. Because the amount of PMMA residue is very small, and the mechanical strength of PMMA is much smaller than that of graphene, there is no influence of

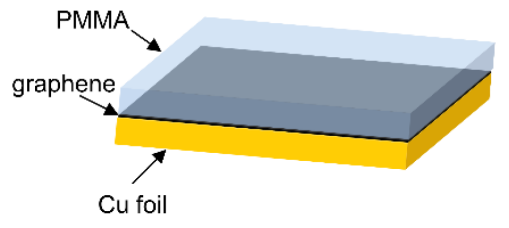

PMMA coating on CVD graphene

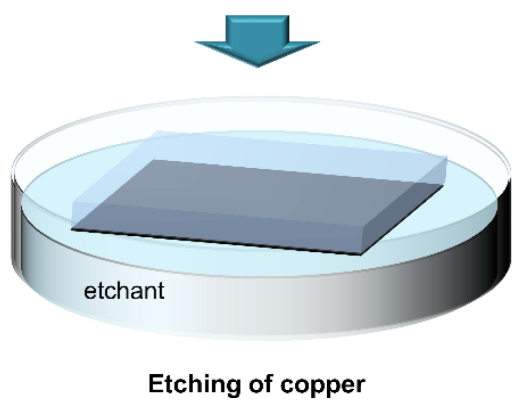

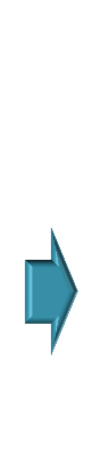

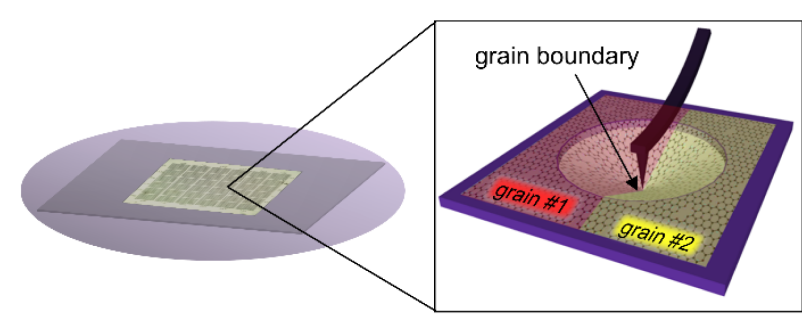

Removal of PMMA

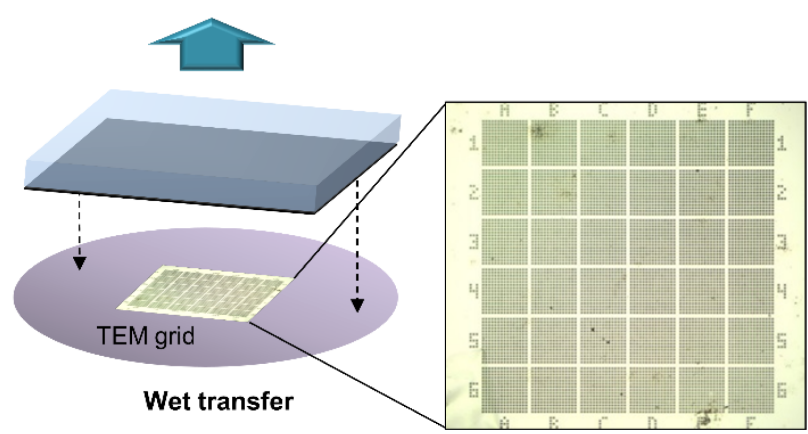

Fig. 1. Schematic of the sample preparation and graphene transfer processes. To minimize the damage of the CVD graphene and TEM grid with 50-nm-thick SiN windows (lower inset), the wet transfer method was employed. The upper inset shows the schematic of the suspended graphene film over a hole of the TEM grid for the AFM nanoindentation test. 
(a)

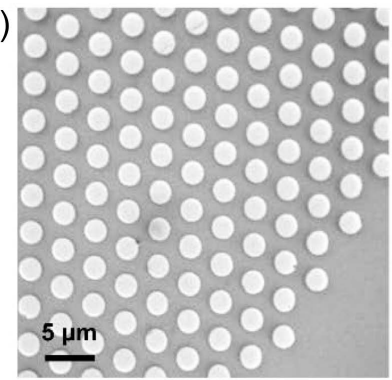

(c)

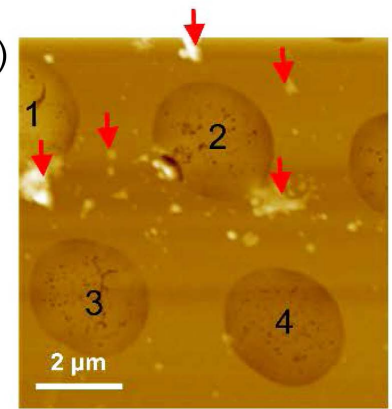

(b)

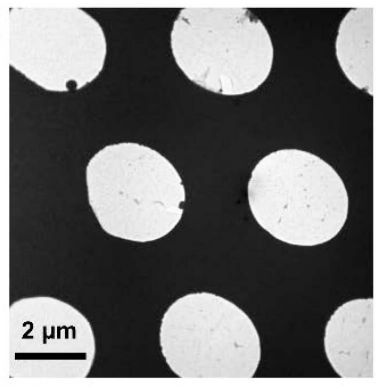

(d)

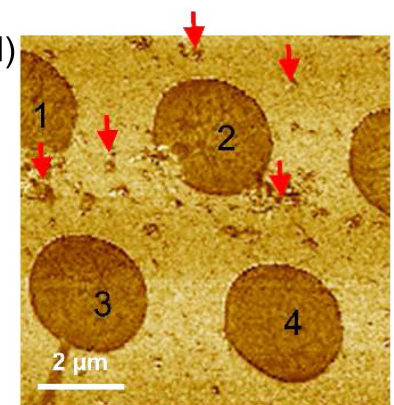

(e)

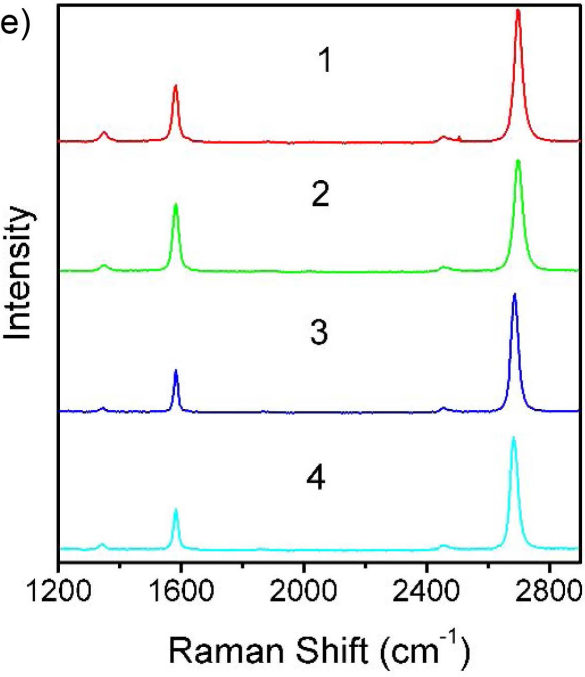

Fig. 2. (a) TEM images of CVD graphene transferred onto a holey TEM grid. In the magnified image, (b), the suspended CVD graphene and PMMA residue can be identified. (c) AFM topography and (d) phase images of the same sample. The red arrows in Figure (c) and (d) indicates the residues of PMMA. Except for them, the transferred CVD graphene film is well suspended over holes without any cracks or damage. (e) Raman spectra obtained from four suspended graphene samples corresponding to the AFM images ((c) and (d)).

PMMA residue on the mechanical test of CVD graphene. It should be noted that the PMMA residue at the GBs enables us to identify the GBs and their exact positions in AFM measurement. It is necessary to directly investigate the mechanical strength of graphene GB, because we can accurately press the GB with a AFM tip.

To confirm the quality of the suspended graphene films,
Raman spectroscopy was employed with a 532-nm laser. As shown in Fig. 2(e), the Raman spectra of four different suspended graphene samples show a relatively high $I_{2 D} / I_{G}$ intensity ratio and negligibly small $\mathrm{D}$ peak, indicating that the transferred CVD graphene is highly crystalline, except for GBs. ${ }^{21,22)}$ The D peak, indicative of defects, appears only in the $\mathrm{GB}$ region. (a)
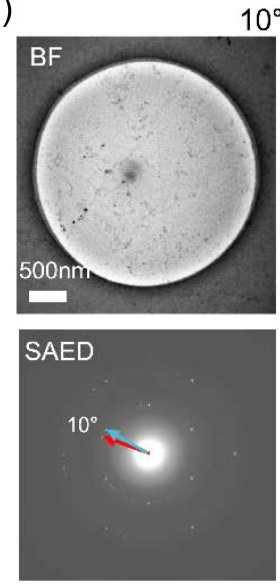

$10^{\circ}-\mathrm{GB}$
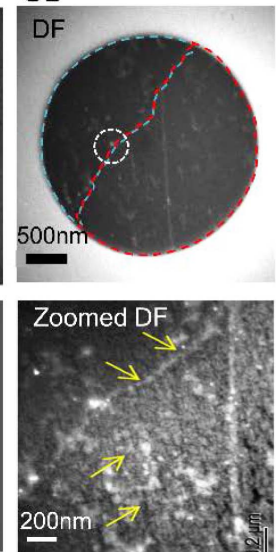

(b)
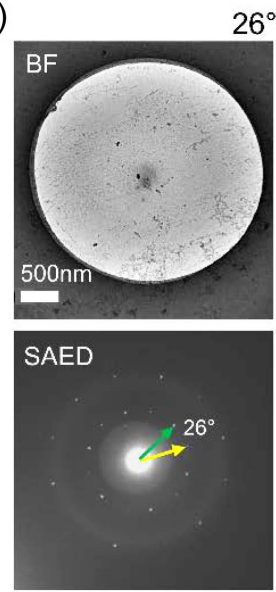

$26^{\circ}-\mathrm{GB}$

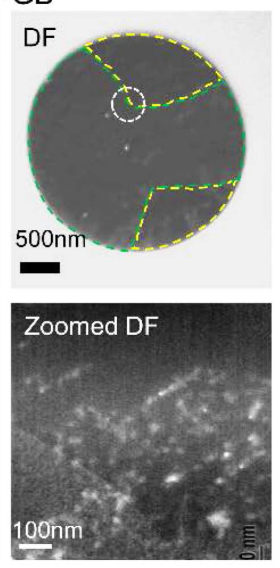

(c)
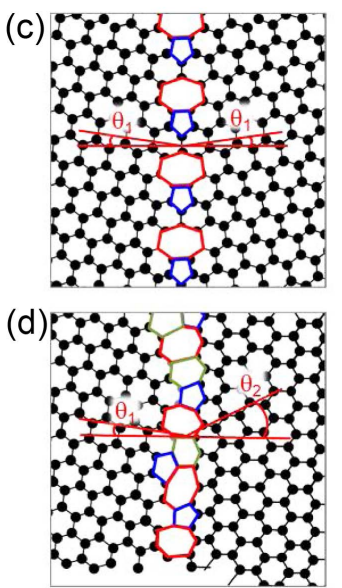

Fig. 3. TEM characterization of the suspended graphene membranes with GB tilt angles of (a) $10^{\circ}$ and (b) $26^{\circ}$. For each sample, $\mathrm{BF}$ and DF images are obtained together with SAED patterns. Each color arrow in the SAED patterns show the crystalline directions of grains corresponding to the grains indicated by the same color borders in the DF images. The measured tilt angles are $10^{\circ}$ and $26^{\circ}$ for (a) and (b), respectively. The magnified DF TEM images obtained from the dash-lined circles in the DF images show the details of the grain boundary structures. As shown by the arrows, the grain boundaries are the combination of straight lines and sudden inflections, and curves, which are similar to a serpentine trail. The schematics of (c) and (d) show atomic structures of grain boundaries used for theoretical simulation in ref. 10 and realistic samples, respectively. 
Next, we observed the distribution of grains and GBs in the transferred CVD graphene. For two separate graphene samples with different tilt angles, we obtained both bright field (BF) and dark field (DF) TEM images, as shown in Fig. 3(a) and (b). Compared with BF TEM images, DF TEM images show more contrast between neighboring grains owing to the different crystalline orientations, which allow for the verification of GBs. The dashed lines in the DF TEM images of Fig. 3(a) and (b) indicate the GBs. From the selected area electron diffraction (SAED) patterns of Fig. 3(a) and (b), we measured the tilt angles of GBs between adjacent graphene grains. If adjacent grains contain symmetrically the same angle of $\theta_{1}$, the tilt angle of the GB is $2 \theta_{1}$ (Fig. 3(c)). In reality, asymmetric GB consisting of pentagon-heptagon rings in CVD graphene can have different crystalline angles of $\theta_{1}$ and $\theta_{2}$ in the left and right lattices. Therefore, the tilt angle of the GB is defined as $\theta_{1}+\theta_{2}$, as shown in Fig. 3(d). From the SAED images, the GBs of Fig. 3(a) and (b) (indicated by dashed circles) yield tilt angles of $10^{\circ}$ and $26^{\circ}$, respectively. Because graphene has a hexagonal structure, the tilt angle can have a range of $0^{\circ}$ to $30^{\circ}$. As shown in the zoomed DF images of Fig. 3(a) and (b), the GBs are not straight, as was claimed in theoretical papers. Although the mechanical strength of graphene GB was theoretically estimated from the straight boundary with a repetitive array of pentagons and heptagons (Fig. 3(c)), the observed GBs of CVD graphene exhibit an irregular array of pentagons and heptagons, and a crooked shape, irrespective of the amplitude of the tilt angle (Fig. 3(d)).

As we previously demonstrated, ${ }^{1,7)}$ the mechanical properties of the suspended CVD graphene films were characterized by the AFM nanoindentation technique. We used a cantilever with an ultrasharp diamond tip (MicroStar Tech, tip radius $=30 \mathrm{~nm}$ ). As demonstrated in Fig. 4, the force-displacement (F-D) curves of CVD graphene samples with various tilt angles were measured by pressing the center of the suspended CVD graphene membrane with the diamond AFM tip until fracture occurred. ${ }^{7)}$ Similar to previous reports, ${ }^{1,7,23)}$ the measured F-D curves were fitted by using Equation (1), where $F, \sigma_{0}{ }^{2 D}, a, \delta$, and $E^{2 \mathrm{D}}$ represent the applied force, pre-tension of the membrane, membrane

(a)

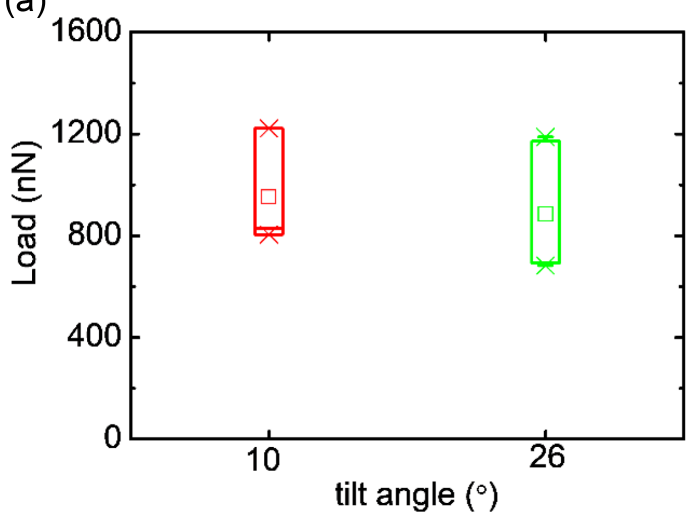

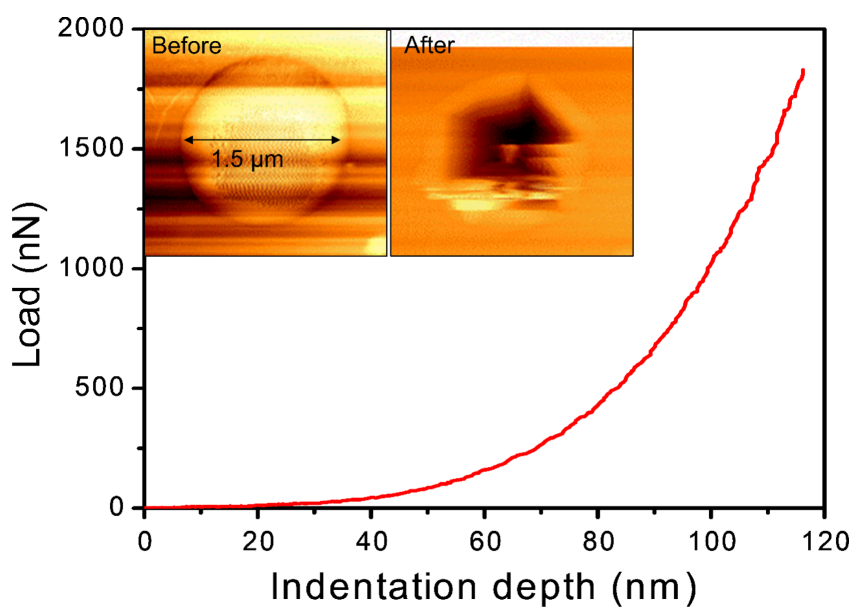

Fig. 4. Representative force-displacement curve of CVD graphene tested by AFM nanoindentation. The insets show the AFM images before and after fracture.

radius, membrane displacement (deflection), and two-dimensional Young's modulus, respectively.

$$
F=\sigma_{0}^{2 D}(\pi a)\left(\frac{\delta}{a}\right)+E^{2 D}\left(q^{3} a\right)\left(\frac{\delta}{a}\right)^{3}
$$

In the formula, $q$ is defined as

$$
q=\frac{1}{1.049-0.15 v-0.16 v^{2}}
$$

where $v$ is the Poisson ratio, equal to the value of graphite ( $v$ $=0.165){ }^{24)}$ No hysteresis was observed during the loading and unloading processes in the F-D curve, indicating that CVD graphene is firmly clamped on the substrate over a hole. Therefore, energy dissipation led by slipping can be neglected. After measuring GB tilt angles in CVD graphene, the AFM tip was located exactly on the GB of the sample. The maximum stress before failure of CVD graphene is called the fracture force. Because GBs can retain high surface activity, its distribution can be easily visualized in the AFM phase. ${ }^{5)}$ After fracture, we can clearly see the fractured graphene over the hole by using AFM (insets in Fig. 4).

(b)

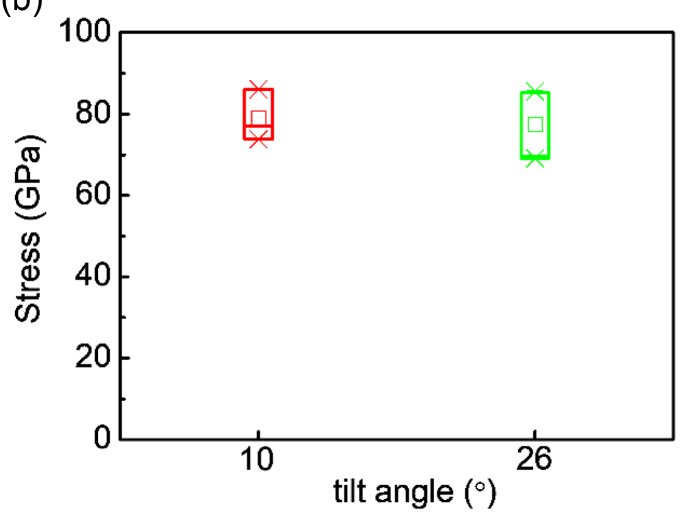

Fig. 5. (a) Fracture loads and (b) fracture strength of the CVD graphene membranes with tilt angles of $10^{\circ}$ and $26^{\circ}$. 
(a)

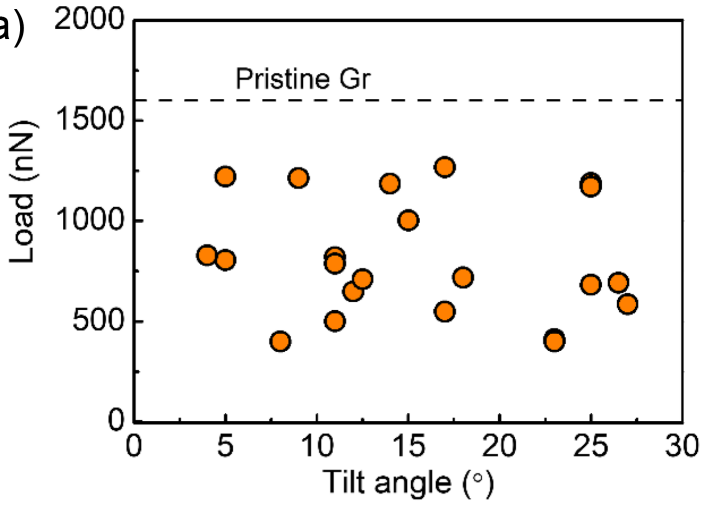

(b)

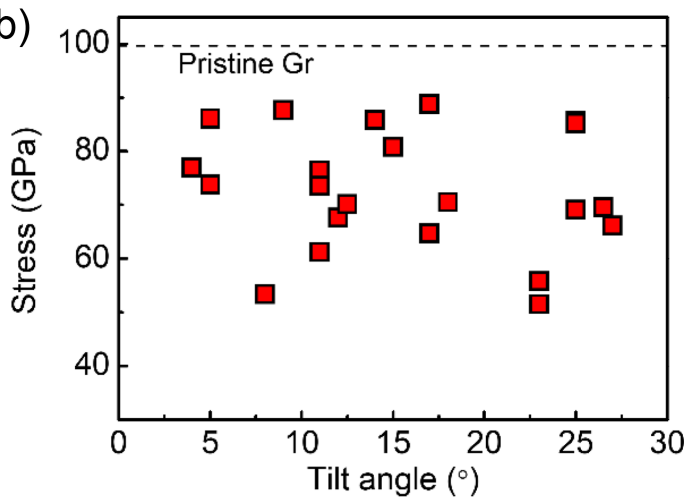

(c)

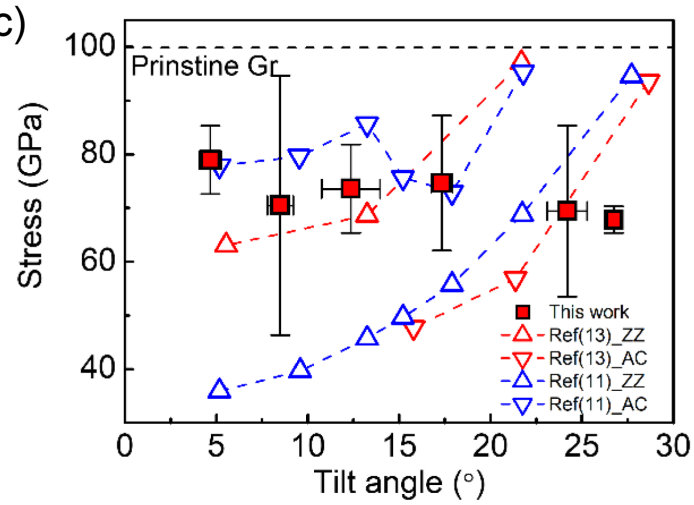

Fig. 6. (a) Experimental measurement of fracture force at the various tilt angles and (b) conversion to 3D true stress. Neither (a) nor (b) shows meaningful angular dependency. (c) Plot of strength against the tilt angle dependence for the graphene GBrelated experiments. The strength of pristine graphene (defect-free) is indicated as a dashed line. The measured strength in our study shows a relatively high strength regardless of tilt angle, consistent with the existing experimental results, ${ }^{7)}$ while other atomistic simulations ${ }^{11,13)}$ showed an increase in strength with tilt angle.

As displayed in Fig. 5(a), the samples with the tilt angles of $10^{\circ}$ and $26^{\circ}$ show similar fracture loads. The mean fracture loads of the samples with GB tilt angles of $10^{\circ}$ and $26^{\circ}$ are $\sim 940$ and $\sim 890 \mathrm{nN}$, respectively. Although the $10^{\circ}$ sample exhibits a slightly higher fracture load value (at most $\sim 10 \%$ smaller than the $26^{\circ}$ sample), both GBs are much stronger than indicated by the previously measured values (higher fracture load by an order of magnitude). ${ }^{5,6)}$ For quantitative analysis, in-plane stresses $\left(\sigma_{\max }^{2 D}\right)$ to break the 2D membrane were measured using the following equa$\operatorname{tion}^{1)}$ :

$$
\sigma_{\max }^{2 D}=\sqrt{\frac{F_{\max } E^{2 D}}{4 \pi a}}
$$

where $F_{\max }$ and $a$ denote the fracture loading force and radius of indenter, respectively. $\mathrm{E}^{2 \mathrm{D}}$ represents the $2 \mathrm{D}$ modulus as in Equation (1). On the basis of our previous finite element method analysis, ${ }^{7}$ we applied the tip radius $(30 \mathrm{~nm})$ in the curve, indicating which values of fracture load meet the $2 \mathrm{D}$ true stress, depending on the tip radius. The thickness of monolayer graphene was assumed to be $0.335 \mathrm{~nm}$ to convert the $2 \mathrm{D}$ values to $3 \mathrm{D}$ values. Consequently, the calculated $\sigma_{\max }{ }^{2 D}$ values for the $10^{\circ}$ and $26^{\circ}$ samples are $\sim 80$ and $\sim 79 \mathrm{GPa}$, respectively, which are comparable to that of pristine graphene ( $100 \mathrm{GPa})$. Moreover, we can conclude that the mechanical strength difference between the $10^{\circ}$ and $26^{\circ}$ samples is not as large as the theoretical prediction. If any, it is likely to be in an allowable experimental error range.

Furthermore, to find out the correlation between the fracture strength and tilt angle, more graphene membranes with various GB tilt angles were studied by TEM and AFM nanoindentation. We summarized all the indentation results as a function of tilt angle, as shown in Fig. 6. The measured fracture loads show highly scattered points in Fig. 6(a) with a broad range of 400-1300 nN. Fig. 6(b) shows that the calculated $\sigma_{\max }{ }^{2 D}$ values have a rather wide window of $\sim 50$ to $\sim 90 \mathrm{GPa}$, regardless of tilt angle. Although the fracture strength of CVD graphene is just a little smaller than that of pristine graphene under biaxial strain conditions, it is worth noting that CVD graphene is still strong. To confirm any relation between the fracture strength and tilt angle, the mean fracture strength values are plotted as a function of tilt angle in Fig. 6(c), where X- and Y-error bars represent the standard deviations. The fracture strength of defect-free graphene (exfoliated one) is indicated by a dashed line. It is clear that the fracture strength of the CVD graphene remains high and insensitive to the tilt angle. Our observa- 
tion of tilt-angle independence of fracture strength is rather inconsistent with the previous atomistic simulation results, which suggest that the fracture strength of graphene increases with tilt angle owing to the relaxation of strain at high tilt-angle GBs. ${ }^{11,13)}$ This discrepancy is attributed to the complicated crystal structure of GBs. The GBs of CVD graphene exhibit random configurations of pentagons, hexagons, and heptagons of distorted structures, as depicted in Fig. 3(d). Such a complicated crystal structure and high distortion can provide more irregular but effective crack propagation paths to minimize the misfit energy, ${ }^{7,25)}$ which has not been reflected in the previous simulations. The crooked shape of grains boundaries, which is composed of an arbitrary mixture of straight lines, curves, and inflection points, eventually eliminates the tilt-angle-dependency of the fracture strength in CVD graphene.

\section{Conclusions}

In conclusion, we demonstrate that the mechanical strength of CVD graphene is just slightly smaller than that of pristine graphene, and there is no tilt-angle-dependence. For samples with GB tilt angles of $10^{\circ}$ and $26^{\circ}$, AFM nanoindentation measurements showed almost the same range of fracture loads. The random and complex inflection points that GBs contain cause the tilt-angle independence. Our study shows that CVD graphene with ultrahigh strength can be produced on a large scale, regardless of graphene crystal orientation or GB type, if GBs are well-stitched with complicated arrangements of pentagon and heptagon carbon rings.

\section{Acknowledgments}

This work was supported by the Korea Institute of Energy Technology Evaluation and Planning (KETEP) and the Ministry of Trade, Industry \& Energy (MOTIE) of the Republic of Korea (No. 20173010013340). The authors also acknowledge support from Samsung Research Funding Center of Samsung Electronics (Project Number SRFC-MA1502-12) and Basic Science Research Program (2016M3A7B4910940)) through the National Research Foundation (NRF) of Korea funded by the Ministry of Science, ICT \& Future Planning.

\section{REFERENCES}

1. C. Lee, X. Wei1, J. W. Kysar, and J. Hone, "Measurement of the Elastic Properties and Intrinsic Strength of Monolayer Graphene," Science, 321 [5887] 385-88 (2008).

2. A. K. Geim and K. S. Novoselov, "The Rise of Graphene," Nat. Mater., 6 183-91 (2007).

3. Y. Gao, S. Kim, S. Zhou, H.-C. Chiu, D. Nélias, C. Berger, W. Heer, L. Polloni, R. Sordan, A. Bongiorno, and E. Riedo, "Elastic Coupling between Layers in Two-Dimensional Materials," Nat. Mater., 14 714-20 (2015).

4. J. H. Kim, J. H. Jeong, N. Kim, R. Joshi, and G.-H. Lee,
"Mechanical Properties of Two-Dimensional Materials and Their Applications,” J. Phys. D: Appl. Phys., 52 [8] 083001 (2018).

5. P. Y. Huang, C. S. Ruiz-Vargas, A. M. Zande, W. S. Whitney, M. P. Levendorf, J. W. Kevek, S. Garg, J. S. Alden, C. J. Hustedt, Y. Zhu, J. Park, P. L. McEuen, and D. A. Muller, "Grains and Grain Boundaries in Single-Layer Graphene Atomic Patchwork Quilts," Nature, 469 389-92 (2011).

6. C. S. Ruiz-Vargas, H. L. Zhuang, P. Y. Huang, A. M. Zande, S. Garg, P. L. McEuen, D. A. Muller, R. G. Hennig, and J. Park, "Softened Elastic Response and Unzipping in Chemical Vapor Deposition Graphene Membranes," Nano Lett., 11 [6] 2259-63 (2011).

7. G.-H. Lee, R. C. Cooper, S. J. An, S. Lee, A. Zande, N. Petrone, A. G. Hammerberg, C. Lee, B. Crawford, W. Oliver, J. W. Kysar, J. Hone, "High-Strength ChemicalVapor-Deposited Graphene and Grain Boundaries," Science, 340 [6136] 1073-76 (2013).

8. X. Li, W. Cai, J. An, S. Kim, J. Nah, D. Yang, R. Piner, A. Velamakanni, I. Jung, E. Tutuc, S. K. Banerjee, L. Colombo, and R. S. Ruoff, "Large-Area Synthesis of High-Quality and Uniform Graphene Films on Copper Foils," Science, 324 [5932] 1312-14 (2009).

9. S. Bae, H. Kim, Y. Lee, X. Xu, J.-S. Park, Y. Zheng, J. Balakrishnan, T. Lei, H. R. Kim, Y. I. Song, Y.-J. Kim, K. S. Kim, B. Özyilmaz, J.-H. Ahn, B. H. Hong, and S. Iijima, "Roll-to-Roll Production of 30-inch Graphene Films for Transparent Electrodes," Nat. Nanotechnol., 5 574-78 (2010).

10. J. C. Meyer, C. Kisielowski, R. Erni, M. D. Rossell, M. F. Crommie, and A. Zettl, "Direct Imaging of Lattice Atoms and Topological Defects in Graphene Membranes," Nano Lett., 8 [11] 3582-86 (2008).

11. Y. Wei, J. Wu, H. Yin, X. Shi, R. Yang, and M. Dresselhaus, "The Nature of Strength Enhancement and Weakening by Pentagon-Heptagon Defects in Graphene," Nat. Mater., 11 759-63 (2012).

12. H. I. Rasool, C. Ophus, W. S. Klug, A. Zettl, and J. K. Gimzewski, "Measurement of the Intrinsic Strength of Crystalline and Polycrystalline Graphene," Nat. Commun., 42811 (2013).

13. R. Grantab, V. B. Shenoy, and R. S. Ruoff, "Anomalous Strength Characteristics of Tilt Grain Boundaries in Graphene," Science, 330 [6006] 946-48 (2010).

14. J. Zhang, J. Zhao, and J. Lu, "Intrinsic Strength and Failure Behaviors of Graphene Grain Boundaries," ACS Nano, 6 [3] 2704-11 (2012).

15. J. W. Suk, R. D. Piner, J. An, and R. S. Ruoff, "Mechanical Properties of Monolayer Graphene Oxide," ACS Nano, 4 [11] 6557-64 (2010).

16. Zhao, J., Z. Guang-Yu, and D.-X. Shi, "Review of GrapheneBased Strain Sensors," Chin. Phys. B, 22 [5] 057701 (2013).

17. Z. Luo, X. Hu, X. Tian, C. Luo, H. Xu, Q. Li, Q. Li, J. Zhang, F. Qiao, X. Wu, V. E. Borisenko, and J. Chu, "Structure-Property Relationships in Graphene-Based Strain and Pressure Sensors for Potential Artificial Intelligence Applications," Sensors, 19 [5] 1250 (2019).

18. P. Y. Huang, C. S. Ruiz-Vargas, A. M. Zande, W. S. Whitney, M. P. Levendorf, J. W. Kevek, S. Garg, J. S. Alden, C. 
J. Hustedt, Y. Zhu, J. Park, P. L. McEuen, and D. A. Muller, "Grains and Grain Boundaries in Single-Layer Graphene Atomic Patchwork Quilts," Nature, 469 [7330] 389-92 (2011).

19. A.W. Tsen, L. Brown, M. P. Levendorf, F. Ghahari, P. Y. Huang, R. W. Havener, C. S. Ruiz-Vargas, D. A. Muller, P. Kim, and J. Park, "Tailoring Electrical Transport across Grain Boundaries in Polycrystalline Graphene,” 336 [6085] 1143-46 (2012).

20. X. Li, M. Sun, C. Shan, Q. Chen, and X. Wei, "Mechanical Properties of 2D Materials Studied by In Situ Microscopy Techniques," Adv. Mater. Interfaces, 5 [5] 1701246.

21. A. C. Ferrari, J. C. Meyer, V. Scardaci, C. Casiraghi, M. Lazzeri, F. Mauri, S. Piscanec, D. Jiang, K. S. Novoselov, S. Roth, and A. K. Geim, "Raman Spectrum of Graphene and Graphene Layers,” Phys. Rev. Lett., 97 [18] 187401
(2006).

22. Y. Hao, Y. Wang, L. Wang, Z. Ni, Z. Wang, R. Wang, C. K. Koo, Z. Shen, and J. T. Thong, "Probing Layer Number and Stacking Order of Few-Layer Graphene by Raman Spectroscopy," Small, 6 [2] 195-200 (2010).

23. K.-T. Wan, S. Guo, and D. A. Dillard, "A Theoretical and Numerical Study of a Thin Clamped Circular Film under an External Load in the Presence of a Tensile Residual Stress," Thin Solid Films, 425 [1] 150-62 (2003).

24. O. L. Blakslee, D. G. Proctor, E. J. Seldin, G. B. Spence, and T. Weng, "Elastic Constants of Compression-Annealed Pyrolytic Graphite,” J. Appl. Phys., 41 [8] 3373-82 (1970).

25. B. Straumal and B. Baretzky, "Grain Boundary Phase Transitions and their Influence on Properties of Polycrystals," Interface Sci., 12 [2] 147-55 (2004). 